Surveyor

\title{
Why was the Circle divided into $360^{\circ}$ ?
}

\section{W Adams}

To cite this article: C. W Adams (1913) Why was the Circle divided into 360? Surveyor, 26:10, 205-208, DOI: 10.1080/18324460.1913.10439094

To link to this article: http://dx.doi.org/10.1080/18324460.1913.10439094

册 Published online: 01 Aug 2012.

Submit your article to this journal 정

LII Article views: 2

Q View related articles ¿ 


\title{
Why was the Circle divided into $360^{\circ}$ ?
}

\author{
By C. W. AdAMS.
}

[This paper was read at the Annual Meeting of the New Zealand Institute of Surveyors, January 7 th, 1913, and published in the "N.Z. Surveyor" of June, 1913.]

I do not suppose that I shall be able to give a satisfactory reply to this question from an historical point of view, as history is not one of my strong points. Still, my own opinion is that as the sun moves round the earth in 365 days, nearly, the ancient astronomers wished to make the number of degrees in a circle agree as nearly as possible with the number of days in a year.

But there are other considerations to be attended to; although Mr. G. J. Morrison in his book on "Mlaps, their Uses and Construction," states on page 6, "There is no reason why 360 should have been chosen rather than 400 , or 100 , or any other number, but it has been used so long that one has come to look upon it as a natural division." Now this statement is quite erroneous, as there are several very good reasons why 360 is much to be preferred to either 100, or 400 . We all know that the chord of $60^{\circ}$ (or the chord of one-sixth of the circumference) is equal to the radíus ; so if we take a pair of compasses and describe any circle, we can divide the circumference into six equal parts, by merely stepping the compasses round the circle. Now, if we divide 100 or 400 by 6 we get a fractional quotient, which, expressed decimally becomes an interminate decimal. There are other reasons, but this one is quite sufficient to cause the rejection of either 100 or 400 as the number of degrees in a circle. 'The French, when they adopted their metric system, divider the circle intn 400 "grades," and spent a large sum of money in calculating tables to suit ; but this system, not being founded on scientific principles, was, of course, soon abandoned.

One chief requisite in the number of divisions in a circle, is that it should contain as many aliquot parts as possible. Now, in general, the larger the number, the more aliquot parts it contains; thus 8 is divisible without remainder by 2 and by 4 only, while $I 2$ is divisible by $2,3,4$, and 6 . But this is not always the case, as in the example of 360 and 400 above mentioned. Still, it was not necessary for the ancients to choose such a high number as 360 ; as the ideal number would have been 240 , which has several special advantages over 360 . Thus 240 is divisible by $2,3,4,5,6,8,10,12,15,16,20,24,30,60$, 80 and 120 . These comprise all the more useful numbers required. Now 360 is divisible by all these numbers except 16 and 80 ; so in this respect 240 is better than 360 . Again, take 360 and 240 and halve them continually, thus :-

$$
\begin{aligned}
& 360,180,90,45,22 \frac{1}{2} \\
& 240,120,60,30,15
\end{aligned}
$$


Now take $r / 5$ of the circle and halve continually,

$$
\begin{aligned}
& 360,72,36,18,9,4 \frac{1}{2} \\
& 240,48,24,12,6,3
\end{aligned}
$$

Now take $\mathrm{x} / 6$ of the circle and halve continually,

$$
\begin{aligned}
& 360,60,30,15,7 \frac{1}{2} \\
& 240,40,20,10,5
\end{aligned}
$$

We thus see that in each of these three examples, 240 can be halved once more than 360 , without producing fractions.

Now let us take the mariner's compass, which divides each quadrant into 8 points, which are again subdivided into quarter points.

$\begin{array}{lll}360^{\circ} & \text { Circumference } & 240^{\circ} \\ 90^{\circ} & \text { Quadrant } & 60^{\circ} \\ 11^{\circ} 15^{\prime} & \text { I point } & 7^{\circ} 30^{\prime} \\ 2^{\circ} 4^{\prime} \frac{3}{4} & 1 \text { point } & 1^{\circ} 52^{\prime} \frac{1}{2} \\ 5^{\circ} 37^{\prime} \frac{1}{2} & \frac{1}{2} \text { point } & 3^{\circ} 45^{\prime} \\ 8^{\circ} 26^{\prime} & \text { 3 point } & 5^{\circ} 37^{\prime} \frac{1}{2} \\ 11^{\circ} \frac{1}{4} & \text { I point } & 7^{\circ} \frac{1}{\frac{1}{2}} \\ 22^{\circ} \frac{1}{2} & 2 \text { points } & 15^{\circ} \\ 33^{\circ} \frac{3}{4} & 3 \text { points } & 22^{\circ} \frac{1}{2} \\ 45^{\circ} & 4 \text { points } & 30^{\circ}\end{array}$

Here, again. we see the greater superiority and simplicity of the $240^{\circ}$ circle. In the 360 division we have $\frac{1}{4}, \frac{1}{2}$ and $\frac{3}{4}$ minutes and $t, \frac{1}{2}$ and 1 degrees; while in the 240 division we have only two halves in the minutes, and two halves in the degrees.

There is another point to be considered. In the introduction to Hutton's Mathematical Tables, there is a Table of Surd expressions for the absolute value of the natural sines of every third degree of the quadrant. This table, as it stands, will serve for the $240^{\circ}$ circle, by applying it to every second clegree, instead of every third. An interesting demonstration of the superiority of the $240^{\circ}$ circle may be obtained from Shortrede's Logarithmic sines and tangents to every second, with the corresponding arguments in time. But we do not get the corresponding arguments in time. We only get one-third of the corresponding arguments in time. Why is this? The answer is in the "additional preface," paragraph 8, where it is stated that "It will be noticed that the time arguments for fractions o a second occur at every third second of space. The two unpressed values are .066 and .133, i.e., repeating decimals that may be viewed as .07 and .13 additive to the preceding tenths. For example, the tabular logs that correspond with $10^{\prime \prime}$ and II", correspond with $.6 \tilde{\gamma}^{\circ}$ and $.73^{8}$. For this reason the proportional parts for seconds of time range from $.01^{\text {s }}$ to $.06^{8}$." This inconvenience is caused, because, while we divide the circle into 24 hours, we foolishly divide it into $360^{\circ}$ instead of $240^{\circ}$. In our present awkward style, one second of arc is equal to I/I5 of a second of time; and as these fifteenths cannot be exactly expressed cxcept by a repeating decimal, they are omitted, but as every $3 / 15$ amounts to $I / 5$, or .2, it is inserted. Now, if we had the more rational division of $240^{\circ}$, then every second of arc would be 
equal to one-tenth of a second of time, and all the time arguments could be shown. Thus :-

\begin{tabular}{|c|c|c|c|c|c|c|c|c|}
\hline \multirow{2}{*}{\multicolumn{2}{|c|}{$\begin{array}{c}\text { Present System } \\
360^{\circ} \\
\mathrm{s}\end{array}$}} & \multicolumn{7}{|c|}{$\begin{array}{l}\text { Proposed Sytsem. } \\
240^{\circ}\end{array}$} \\
\hline & & \multirow{3}{*}{$\begin{array}{l}.00 \\
.10\end{array}$} & & \multirow{2}{*}{\multicolumn{5}{|c|}{ Proportional parts. }} \\
\hline \multirow[t]{3}{*}{.00} & \multirow{2}{*}{$\begin{array}{l}\text { oo } \\
\text { or }\end{array}$} & & \multirow{2}{*}{ oo } & & & & & \\
\hline & & & & \multicolumn{4}{|c|}{$32^{\circ} \quad 16^{\prime}$} & \\
\hline & 02 & .20 & 02 & $S$ & & $\mathrm{~s}$ & $\mathrm{~T}$ & $\mathrm{C}$ \\
\hline \multirow[t]{3}{*}{.20} & 03 & .30 & 03 & .or & .I & 5 & 7 & 2 \\
\hline & 04 & .40 & 04 & .02 & .2 & ro & $I_{4}$ & 4 \\
\hline & 05 & .50 & 05 & .03 & .3 & I5 & $2 I$ & 6 \\
\hline \multirow[t]{3}{*}{.40} & 06 & .60 & 06 & .04 & .4 & 20 & 28 & 8 \\
\hline & 07 & .70 & 07 & .05 & .5 & 25 & 35 & Io \\
\hline & 08 & .80 & 08 & .06 & .6 & 30 & 42 & I2 \\
\hline \multirow[t]{3}{*}{.60} & 09 & .90 & 09 & .07 & .7 & 35 & 49 & I4 \\
\hline & Io & 1.00 & Io & .08 & .8 & 40 & 56 & 16 \\
\hline & II & I.IO & II & .09 & .9 & 45 & 63 & I8 \\
\hline \multirow[t]{3}{*}{.80} & 12 & 1.20 & 12 & .10 & r.o & 50 & 70 & 20 \\
\hline & I3 & 1.30 & I3 & & & & & \\
\hline & $\mathrm{r} 4$ & I.40 & I4 & & $57^{\circ}$ & $43^{\prime}$ & & \\
\hline I.00 & 15 & I. $5^{\circ}$ & 15 & & & & & \\
\hline
\end{tabular}

The tables of porportional parts would also be much simplified as one table only would be required for arguments both in arc and time, as shown above. We see also, that under the present system, the proportionate parts for time and space do not apply to equal increments. For instance, if we look at the proportionate parts in Shortrede's logs, :we see that they apply to tenths of seconds of arc. and to hundredths of seconds of time. But the tenth of a second of arc is only $2 / 3$ of the hundredth of a second of time, therefore we have 9 proportionate parts for seconds of arc, and only 6 proportionate parts for seconds of time. This is another anomaly that would be done away with if we had a $240^{\circ}$ circle.

Another example:-In the large table of Natural Haversines, edited by Major General Hannyngton, F.R.A.S., the haversines are given to seven places of decimals, and for every 10 " for the whole circle. Each page contains one degree, or I8o pages in all. From $0^{\circ}$ to $I 80^{\circ}$, the degrees are printed in large type at the head of each page, and from $180^{\circ}$ to $360^{\circ}$, the degrees are printed at the bottom of each page. There are very convenient tables of proportional parts, for seconds of arc, and tenths of seconds, so the haversine of any arc to seven places of decimals, and to the nearest tenth of a second, can be taken out almost at a glance.

The time equivalents are given as well for every $\left(I^{\prime}\right)$ minute of arc, down the left side of the page, $4^{\mathrm{s}}$ being the time equivalent. And also for each 10" of arc along the top of the page, but as

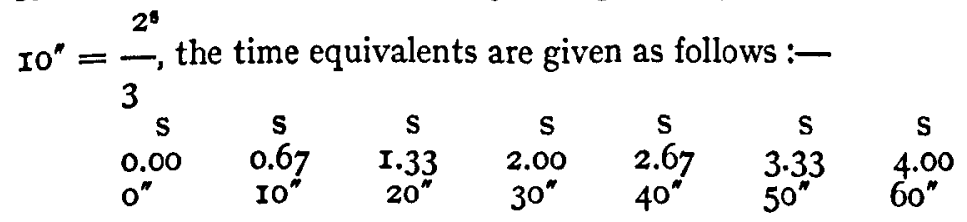


Here, again, we see how very dissimilar the arc and time equiva lents are, but if we adopt the $240^{\circ}$ circle, the time eqiuvalents would be as follows :-

$\begin{array}{crrrrrr}S & S & S & S & S & S & S \\ 0.0 & I & 2 & 3 & 4 & 5 & 6 \\ 0^{\prime \prime} & \mathrm{IO}^{\prime} & 20^{\prime \prime} & 30^{\prime \prime} & 40^{\prime \prime} & 50^{\prime \prime} & 60^{\prime \prime}\end{array}$

But although the tables of proportional parts are most convenient for seconds and tenths of arc, they cannot be used at all for decimals of a second of time, whereas with a $240^{\circ}$ circle the same table of proportional parts would serve for tenths and hundredths of a second of time; as well as for seconds and tenths of arc. To sum up :

Under our present system $15^{\circ}=I$ hour ; $I 5^{\prime}=I$ min. ; and $15^{\prime \prime}$ $=I$ sec., also 4 secs. time $=I^{\prime} ; 4 \mathrm{~min} .=I^{\circ} ;$ and 6 hours $=I$ quadrant, or $90^{\circ}$.

Under the proposed system, $240^{\circ}=24$ hours; $10^{\circ}=\mathrm{I}$ hour ; $I 0^{\prime}=I$ min.; and $I 0^{\prime \prime}=I$ sec. Also 6 secs time $=I^{\prime} ; 6$ min. $=$ $I^{\circ} ;$ and 6 hours $=I$ quadrant, or 60 degrees. So to convert time into arc, we multiply the hours, minutes and seconds by ro; and to convert arc into time, we divide the ${ }^{\circ}, '$ and " by 10.

The same complicated and confusing method of porportional parts that we meet with in Shortrede's tables, and also in Major Hannyngton's tables of haversines, must also obtain in all tables of trig. functions under our present system, whenever it is attempted to give " arguments " or proportional parts for less quantities than I5" of arc, or I second of time. Now, if the French Savants had only thought of the brilliant idea put forth in this article, and had adopted the $240^{\circ}$ circle instead of the $400^{\circ}$ grade circle, they would have introduced a splendid reform, and covered themselves with glory! Perhaps it is not yet too late to make the change!

\section{Protection of Coast Lines.}

Readers of general literature will know that in recent years considerable attention has been diverted to enquiries concerning the stability of the British coast line, a general fear having seized the nation that the sea was bent on swallowing up the little island which had for centuries claimed the proud privilege of ruling that element. There were instances enough of encroachment to cause alarm, the most notable perhaps being that of Dunwich, a prosperous town of 40,00 inhabitants, between Southwold and Aldeburgh, on the coast of Suffolk, which had been swallowed up whole, while numerous minor cases constantly occurred along the friable cliffs of the east and south coasts. The general alarm led to the appointment in Igo6 of a Royal Commission, which enquired into the whole question, and happily came to the conclusion that such erosion as was proceeding was balanced by natural reclamation, and the net result was that the British Isles today covered as much dry land on the earth's surface as they had ever 